Physics of Plasmas 13 (2006) 052107

\title{
Magnetohydrodynamics of Fractal Media
}

\author{
Vasily E. Tarasov \\ Skobeltsyn Institute of Nuclear Physics, \\ Moscow State University, Moscow 119991, Russia*
}

\begin{abstract}
The fractal distribution of charged particles is considered. An example of this distribution is the charged particles that are distributed over fractal. The fractional integrals are used to describe fractal distribution. These integrals are considered as approximations of integrals on fractals. Typical turbulent media could be of a fractal structure and the corresponding equations should be changed to include the fractal features of the media. The magnetohydrodynamics equations for fractal media are derived from the fractional generalization of integral Maxwell equations and integral hydrodynamics (balance) equations. Possible equilibrium states for these equations are considered.
\end{abstract}

\section{INTRODUCTION}

The theory of integrals and derivatives of noninteger order goes back to Leibniz, Liouville, Riemann, Grunwald, and Letnikov [1, 2]. Fractional analysis has found many applications in recent studies in mechanics and physics. The interest in fractional integrals and derivatives has been growing continually during the last few years because of numerous applications. In a fairly short period of time the list of such applications becomes long, and include chaotic dynamics [3, 4], material sciences [5, 6, 7, 8], mechanics of fractal and complex media [9, 10, 11, 12], quantum mechanics [13, 14], physical kinetics [3, 15, 16, 17], plasma physics [18, 21], electromagnetic theory [19, 20, 21], astrophysics [22], long-range dissipation [23, 24], non-Hamiltonian mechanics [25, 26], long-range interaction [27, 28], anomalous diffusion, and transport theory [3, 29, 30, 31]].

${ }^{*}$ Electronic address: E-mail: tarasov@theory.sinp.msu.ru 
The new type of problem has increased rapidly in areas in which the fractal features of a process or the medium impose the necessity of using nontraditional tools in "regular" smooth physical equations. To use fractional derivatives and fractional integrals for fractal distribution, we must use some continuous model [10,11]. We propose to describe the fractal medium by a fractional continuous model [10], where all characteristics and fields are defined everywhere in the volume, but they follow some generalized equations that are derived by using fractional integrals. In many problems the real fractal structure of the medium can be disregarded and the fractal medium can be replaced by some fractional continuous mathematical model. Smoothing of microscopic characteristics over the physically infinitesimal volume transforms the initial fractal medium into the fractional continuous model [10, 11] that uses the fractional integrals. The order of the fractional integral is equal to the fractal dimension of distribution. The fractional integrals allow us to take into account the fractality of the distribution. Fractional integrals can be considered as approximations of integrals on fractals [38, 39]. In Ref. [38, 39], authors proved that integrals on fractals can be approximated by fractional integrals. In Ref. [25], we proved that fractional integrals can be considered as integrals over the space with a fractional dimension up to the numerical factor.

The distribution on the fractal can be described by a fractional continuous medium model [10, 11, 12, 21]. In the general case, the fractal medium cannot be considered as a continuous medium. There are points and domains without particles. In Refs. [10, 11, 21], we suggest considering the fractal distributions as special (fractional) continuous media. We use the procedure of replacement of the distribution with fractal mass dimension by some continuous model that uses fractional integrals. This procedure is a fractional generalization of the Christensen approach [41]. The suggested procedure leads to the fractional integration to describe the fractal medium. In this paper, we consider the magnetohydrodynamics equations for the fractal distribution of charged particles. Note that typical turbulent media could be of a fractal structure and the corresponding equations should be changed to include the fractal features of the media.

In Sec. II, a brief review of the Hausdorff measure, Hausdorff dimension and integration on fractals is suggested to fix notation and provide a convenient reference. The connection integration on fractals and fractional integration is discussed. In Sec. III, a brief review of electrodynamics of the fractal distribution of charged particles is given. The densities 
of electric charge and current for the fractal distribution are described. A fractional generalization of the integral Maxwell equation is suggested. In Sec. IV, a brief review of the hydrodynamics of fractal media is considered to fix notation and provide a convenient reference. In Sec. V, the magnetohydrodynamics equations for the fractal distribution of charged particles are derived. The stationary states for these equations are considered. Finally, a short conclusion is given in Sec. VI.

\section{INTEGRATION ON FRACTAL AND FRACTIONAL INTEGRATION}

Fractals are measurable metric sets with a noninteger Hausdorff dimension. The main property of the fractal is noninteger Hausdorff dimension. Let us consider a brief review of the Hausdorff measure and the Hausdorff dimension [35, 40] to fix the notation and provide a convenient reference.

\section{A. Hausdorff measure and Hausdorff dimension}

Consider a measurable metric set $\left(W, \mu_{H}\right)$. The elements of $W$ are denoted by $x, y, z, \ldots$, and represented by $n$-tuples of real numbers, $x=\left(x_{1}, x_{2}, \ldots, x_{n}\right)$, such that $W$ is embedded in $\mathbb{R}^{n}$. The set $W$ is restricted by the following conditions: (1) $W$ is closed; (2) $W$ is unbounded; (3) $W$ is regular (homogeneous, uniform) with its points randomly distributed.

The metric $d(x, y)$ as a function of two points $x$ and $y \in W$ can be defined by

$$
d(x, y)=\sum_{i=1}^{n}\left|y_{i}-x_{i}\right|
$$

or

$$
d(x, y)=|x-y|=\left(\sum_{i=1}^{n}\left(y_{i}-x_{i}\right)^{2}\right)^{1 / 2} .
$$

The diameter of a subset $E \subset W \subset \mathbb{R}^{n}$ is

$$
d(E)=\operatorname{diam}(E)=\sup \{d(x, y): x, y \in E\},
$$

Let us consider a set $\left\{E_{i}\right\}$ of non-empty subsets $E_{i}$ such that $\operatorname{dim}\left(E_{i}\right)<\varepsilon, \forall i$, and $W \subset \bigcup_{i=1}^{\infty} E_{i}$. Then, we define

$$
\xi\left(E_{i}, D\right)=\omega(D)\left[\operatorname{diam}\left(E_{i}\right)\right]^{D}=\omega(D)\left[d\left(E_{i}\right)\right]^{D} .
$$


The factor $\omega(D)$ depends on the geometry of $E_{i}$, used for covering $W$. If $\left\{E_{i}\right\}$ is the set of all (closed or open) balls in $W$, then

$$
\omega(D)=\frac{\pi^{D / 2} 2^{-D}}{\Gamma(D / 2+1)} .
$$

The Hausdorff dimension $D$ of a subset $E \subset W$ is defined [32, 33, 34, 35] by

$$
D=\operatorname{dim}_{H}(E)=\sup \left\{d \in R: \quad \mu_{H}(E, d)=\infty\right\}
$$

or

$$
D=\operatorname{dim}_{H}(E)=\inf \left\{d \in R: \quad \mu_{H}(E, d)=0\right\}
$$

From (5) and (6), we obtain

1) $\mu_{H}(E, d)=0$ for $d>D=\operatorname{dim}_{H}(E)$;

2) $\mu_{H}(E, d)=\infty$ for $d<D=\operatorname{dim}_{H}(E)$.

The Hausdorff measure $\mu_{H}$ of a subset $E \subset W$ is defined [32, 33, 34, 35] by

$$
\mu_{H}(E, D)=\lim _{\varepsilon \rightarrow 0} \inf _{\left\{E_{i}\right\}}\left\{\sum_{i=1}^{\infty} \xi\left(E_{i}, D\right): \quad E \subset \bigcup_{i} E_{i}, \quad d\left(E_{i}\right)<\varepsilon \quad \forall i\right\},
$$

or

$$
\mu_{H}(E, D)=\omega(D) \lim _{d\left(E_{i}\right) \rightarrow 0} \inf _{\left\{E_{i}\right\}} \sum_{i=1}^{\infty}\left[d\left(E_{i}\right)\right]^{D} .
$$

If $E \subset W$ and $\lambda>0$, then

$$
\mu_{H}(\lambda E, D)=\lambda^{D} \mu_{H}(E, D)
$$

where $\lambda E=\{\lambda x, x \in E\}$.

\section{B. Function and integrals on fractal}

Let us consider the functions on $W$ :

$$
f(x)=\sum_{i=1}^{\infty} \beta_{i} \chi_{E_{i}}(x)
$$

where $\chi_{E}$ is the characteristic function of $E: \chi_{E}(x)=1$ if $x \in E$, and $\chi_{E}(x)=0$ if $x \notin E$. For continuous function $f(x)$ :

$$
\lim _{x \rightarrow y} f(x)=f(y)
$$


whenever

$$
\lim _{x \rightarrow y} d(x, y)=0 .
$$

The Lebesgue-Stieltjes integral for (9) is defined by

$$
\int_{W} f d \mu=\sum_{i=1}^{\infty} \beta_{i} \mu_{H}\left(E_{i}\right) .
$$

Therefore

$$
\begin{gathered}
\int_{W} f(x) d \mu_{H}(x)=\lim _{d\left(E_{i}\right) \rightarrow 0} \sum_{E_{i}} f\left(x_{i}\right) \xi\left(E_{i}, D\right)= \\
=\omega(D) \lim _{d\left(E_{i}\right) \rightarrow 0} \sum_{E_{i}} f\left(x_{i}\right)\left[d\left(E_{i}\right)\right]^{D} .
\end{gathered}
$$

It is always possible to divide $\mathbb{R}^{n}$ into parallelepipeds:

$$
E_{i_{1} \ldots i_{n}}=\left\{\left(x_{1}, \ldots, x_{n}\right) \in W: x_{j}=\left(i_{j}-1\right) \Delta x_{j}+\alpha_{j}, 0 \leq \alpha_{j} \leq \Delta x_{j}, \quad j=1, \ldots, n\right\}
$$

Then

$$
\begin{gathered}
d \mu_{H}(x)=\lim _{d\left(E_{i_{1} \ldots i_{n}}\right) \rightarrow 0} \xi\left(E_{i_{1} \ldots i_{n}}, D\right)= \\
=\lim _{d\left(E_{i_{1} \ldots i_{n}}\right) \rightarrow 0} \prod_{j=1}^{n}\left(\Delta x_{j}\right)^{D / n}=\prod_{j=1}^{n} d^{D / n} x_{j} .
\end{gathered}
$$

The range of integration $W$ may also be parametrized by polar coordinates with $r=d(x, 0)$ and angle $\Omega$. Then $E_{r, \Omega}$ can be thought of as spherically symmetric covering around a center at the origin. In the limit, the function $\xi\left(E_{r, \Omega}, D\right)$ gives

$$
d \mu_{H}(r, \Omega)=\lim _{d\left(E_{r, \Omega}\right) \rightarrow 0} \xi\left(E_{r, \Omega}, D\right)=d \Omega^{D-1} r^{D-1} d r
$$

Let us consider $f(x)$ that is symmetric with respect to some center $x_{0} \in W$, i.e., $f(x)=$ const for all $x$, such that $d\left(x, x_{0}\right)=r$ for arbitrary values of $r$. Then the transformation

$$
W \rightarrow W^{\prime}: x \rightarrow x^{\prime}=x-x_{0}
$$

can be performed to shift the center of symmetry. Since $W$ is not a linear space, (17) need not be a map of $W$ onto itself; (17) is measure preserving. Then the integral over a $D$-dimensional metric space is

$$
\int_{W} f d \mu_{H}=\lambda(D) \int_{0}^{\infty} f(r) r^{D-1} d r
$$


where

$$
\lambda(D)=\frac{2 \pi^{D / 2}}{\Gamma(D / 2)} .
$$

This integral is known in the theory of the fractional calculus [1]. The right RiemannLiouville fractional integral is

$$
\left(I_{-}^{D} f\right)(z)=\frac{1}{\Gamma(D)} \int_{z}^{\infty}(x-z)^{D-1} f(x) d x
$$

For $z=0$, Eq. (20) gives

$$
\left(I_{-}^{D} f\right)(0)=\frac{1}{\Gamma(D)} \int_{0}^{\infty} x^{D-1} f(x) d x,
$$

and Eq. (18) is reproduced by

$$
\int_{W} f d \mu_{H}=\frac{2 \pi^{D / 2} \Gamma(D)}{\Gamma(D / 2)}\left(I_{-}^{D} f\right)(0) .
$$

Equation (22) connects the integral on fractal with an integral of fractional order. This result permits us to apply different tools of the fractional calculus [1] for the fractal medium. As a result, the fractional integral can be considered as an integral on the fractal up to the numerical factor $\Gamma(D / 2) /\left[2 \pi^{D / 2} \Gamma(D)\right]$.

Note that the interpretation of fractional integration is connected with a fractional dimension [25]. This interpretation follows from the well-known formulas for dimensional regularization [37]. The fractional integral can be considered as an integral in the fractional dimension space up to the numerical factor $\Gamma(D / 2) /\left[2 \pi^{D / 2} \Gamma(D)\right]$. In Ref. [38] it was proved that the fractal space-time approach is technically identical to the dimensional regularization.

\section{Properties of integrals}

The integral defined in (13) satisfies the following properties:

(1) Linearity:

$$
\int_{W}\left(a f_{1}+b f_{2}\right) d \mu_{H}=a \int_{W} f_{1} d \mu_{H}+b \int_{W} f_{2} d \mu_{H}
$$

where $f_{1}$ and $f_{2}$ are arbitrary functions; $a$ and $b$ are arbitrary constants.

(2) Translational invariance:

$$
\int_{W} f\left(x+x_{0}\right) d \mu_{H}(x)=\int_{W} f(x) d \mu_{H}(x)
$$


since $d \mu_{H}\left(x-x_{0}\right)=d \mu_{H}(x)$ as a consequence of homogeneity (uniformity).

(3) Scaling property:

$$
\int_{W} f(\lambda x) d \mu_{H}(x)=\lambda^{-D} \int_{W} f(x) d \mu_{H}(x)
$$

since $d \mu_{H}(x / \lambda)=\lambda^{-D} d \mu_{H}(x)$.

By evaluating the integral of the function $f(x)=\exp \left(-a x^{2}+b x\right)$, it has been shown [36, 37] that conditions (23)-(25) define the integral up to normalization:

$$
\int_{W} \exp \left(-a x^{2}+b x\right) d \mu_{H}(x)=\pi^{D / 2} a^{-D / 2} \exp \left(b^{2} / 4 a\right) .
$$

Note that, for $b=0$, Eq. (26) is identical to result from (22), which can be obtained directly without conditions (223)-(25).

\section{Multi-variable integration on fractal}

The integral in (18) is defined for a single variable. It is only useful for integrating spherically symmetric functions. We consider multiple variables by using the product spaces and product measures.

Let us consider a collection of $n=3$ measurable sets $\left(W_{k}, \mu_{k}, D\right)$ with $k=1,2,3$, and form a Cartesian product of the sets $W_{k}$ producing $W=W_{1} \times W_{2} \times W_{3}$. The definition of product measures and the application of the Fubinis theorem provides a measure for the product set $W=W_{1} \times W_{2} \times W_{3}$ as

$$
\left(\mu_{1} \times \mu_{2} \times \mu_{3}\right)(W)=\mu_{1}\left(W_{1}\right) \mu_{2}\left(W_{2}\right) \mu_{3}\left(W_{3}\right) .
$$

Then integration over a function $f$ on $W$ is

$$
\begin{gathered}
\int_{W} f\left(x_{1}, x_{2}, x_{3}\right) d\left(\mu_{1} \times \mu_{2} \times \mu_{3}\right)= \\
=\int_{W_{1}} \int_{W_{2}} \int_{W_{3}} f\left(x_{1}, x_{2}, x_{3}\right) d \mu_{1}\left(x_{1}\right) d \mu_{2}\left(x_{2}\right) d \mu_{3}\left(x_{3}\right) .
\end{gathered}
$$

In this form, the single-variable measure from (18) may be used for each coordinate $x_{k}$, which has an associated dimension $\alpha_{k}$ :

$$
d \mu_{k}\left(x_{k}\right)=\lambda\left(\alpha_{k}\right)\left|x_{k}\right|^{\alpha_{k}-1} d x_{k}, \quad k=1,2,3 .
$$


Then the total dimension of $W=W_{1} \times W_{2} \times W_{3}$ is

$$
D=\alpha_{1}+\alpha_{2}+\alpha_{3}
$$

Let us reproduce the result for the single-variable integration (18), from $W_{1} \times W_{2} \times W_{3}$. We take a spherically symmetric function $f\left(x_{1}, x_{2}, x_{3}\right)=f(r)$, where $r^{2}=\left(x_{1}\right)^{2}+\left(x_{2}\right)^{2}+\left(x_{3}\right)^{2}$ and perform the integration in spherical coordinates $(r, \phi, \theta)$. Equation (28) becomes

$$
\begin{gathered}
\int_{W} d \mu_{1}\left(x_{1}\right) d \mu_{2}\left(x_{2}\right) d \mu_{3}\left(x_{3}\right) f\left(x_{1}, x_{2}, x_{3}\right)= \\
=A(\alpha) \int_{W_{1}} d x_{1} \int_{W_{2}} d x_{2} \int_{W_{3}} d x_{3}\left|x_{1}\right|^{\alpha_{1}-1}\left|x_{2}\right|^{\alpha_{2}-1}\left|x_{3}\right|^{\alpha_{3}-1} f\left(x_{1}, x_{2}, x_{3}\right)= \\
=A(\alpha) \int d r \int d \phi \int d \theta J_{3} r^{\alpha_{1}+\alpha_{2}+\alpha_{3}-3}(\cos \phi)^{\alpha_{1}-1}(\sin \phi)^{\alpha_{2}+\alpha_{3}-2}(\sin \theta)^{\alpha_{3}-1} f(r),
\end{gathered}
$$

where $J_{3}=r^{2} \sin \phi$ is the Jacobian of the coordinate change, and $A(\alpha)=\lambda\left(\alpha_{1}\right) \lambda\left(\alpha_{2}\right) \lambda\left(\alpha_{3}\right)$. Since the function is only dependent on the radial variable and not the angular variables, we can use

$$
\int_{0}^{\pi / 2} \sin ^{\mu-1} x \cos ^{\nu-1} x d x=\frac{\Gamma(\mu / 2) \Gamma(\nu / 2)}{2 \Gamma((\mu+\nu) / 2)} .
$$

where $\mu>0, \nu>0$. From (30), we obtain

$$
\int_{W} d \mu_{1}\left(x_{1}\right) d \mu_{2}\left(x_{2}\right) d \mu_{3}\left(x_{3}\right) f(r)=\lambda(D) \int f(r) r^{D-1} d r,
$$

where

$$
\lambda(D)=\frac{2 \pi^{D / 2}}{\Gamma(D / 2)} .
$$

This equation describes the $D$-dimensional integration [37] of a spherically symmetric function, and reproduces the result (18).

\section{E. Density function and mass on fractal}

Let us consider the mass that is distributed on the measurable metric set $W$ with the fractional Hausdorff dimension $D$. Suppose that the density of mass distribution is described by the function $\rho(\mathbf{r})$ that is defined by (9). In this case, the mass can be derived by

$$
M_{D}(W)=\int_{W} \rho(\mathbf{r}) d V_{D}
$$

where

$$
d V_{D}=d \mu_{1}\left(x_{1}\right) d \mu_{2}\left(x_{2}\right) d \mu_{3}\left(x_{3}\right)=c_{3}(D, \mathbf{r}) d x d y d z
$$




$$
\begin{gathered}
c_{3}(D, \mathbf{r})=\lambda\left(\alpha_{1}\right) \lambda\left(\alpha_{2}\right) \lambda\left(\alpha_{3}\right) x^{\alpha_{1}-1} y^{\alpha_{2}-1} z^{\alpha_{3}-1}, \\
\operatorname{dim}_{H}(W)=D=\alpha_{1}+\alpha_{2}+\alpha_{3} .
\end{gathered}
$$

As a result, we have

$$
M_{D}(W)=\int_{W} \rho(\mathbf{r}) d V_{D}, \quad d V_{D}=c_{3}(D, \mathbf{r}) d V_{3},
$$

where $d V_{3}=d x d y d z$ for Cartesian coordinates, and

$$
c_{3}(D, \mathbf{r})=\frac{8 \pi^{D / 2}|x|^{\alpha_{1}-1}|y|^{\alpha_{2}-1}|z|^{\alpha_{3}-1}}{\Gamma\left(\alpha_{1}\right) \Gamma\left(\alpha_{2}\right) \Gamma\left(\alpha_{3}\right)} .
$$

As a result, we get the Riemann-Liouville fractional integral [1] up to a numerical factor $8 \pi^{D / 2}$.

\section{F. Mass of fractal distribution}

The cornerstone of fractals is the noninteger dimension. The fractal dimension can be best calculated by box counting method, which means drawing a box of size $R$ and counting the mass inside. This mass fractal dimension can be easy measured for fractal media. The properties of the fractal medium like mass obey a power law relation $M \sim R^{D}$, where $M$ is the mass of the fractal medium, $R$ is a box size (or a sphere radius), and $D$ is a mass fractal dimension. The power law relation $M \sim R^{D}$ can be naturally derived by using the fractional integral [10]. The mass fractal dimension is connected [10] with the order of fractional integrals.

Consider the region $W$ in three-dimensional Euclidean space $\mathbb{R}^{3}$. The volume of the region $W$ is denoted by $V_{D}(W)$. The mass of the region $W$ in the fractal medium is denoted by $M_{D}(W)$. The fractality of the medium means than the mass of this medium in any region $W$ of Euclidean space $\mathbb{R}^{3}$ increases more slowly than the volume of this region. For the ball region of the fractal medium, this property can be described by the power law $M \sim R^{D}$, where $R$ is the radius of the ball $W$.

The fractal medium is called a homogeneous one if the power law $M \sim R^{D}$ does not depend on the translation of the region. The homogeneity property of the medium can be formulated in the form: For all regions $W$ and $W^{\prime}$ of the homogeneous fractal medium with the equal volumes $V_{D}(W)=V_{D}\left(W^{\prime}\right)$, the masses of these regions are equal $M_{D}(W)=$ $M_{D}\left(W^{\prime}\right)$. Note that the wide class of the fractal media satisfies the homogeneous property. 
In Refs. [10], the continuous medium model for the fractal media was suggested. The fractality and homogeneity properties can be realized in the following forms: (1) Homogeneity: The local density of homogeneous fractal media is a translation invariant value that has the form $\rho(\mathbf{r})=\rho_{0}=$ const. (2) Fractality: The mass of the ball region $W$ of a fractal medium obeys a power law relation, $M \sim R^{D}$, where $0<D<3$, and $R$ is the radius of the ball. These requirements can be realized by the fractional generalization (39) of the equation

$$
M_{3}(W)=\int_{W} \rho(\mathbf{r}) d V_{3} .
$$

The form of function $c_{3}(D, \mathbf{r})$ is defined by the properties of the fractal medium. Note that the final equations that relate the physical variables have a form that is independent of a numerical factor in the function $c_{3}(D, \mathbf{r})$. However, the dependence of $\mathbf{r}$ is important to these equations.

Equation (39) describes the mass that is distributed in the volume and has the mass fractal dimension $D$ by fractional integrals. There are many different definitions of fractional integrals [1]. The fractional integrals can be used to describe fields that are defined on the set $W$ with fractional Hausdorff dimension $\operatorname{dim}_{H}(W)=D$.

For the Riemann-Liouville fractional integral,

$$
c_{3}(D, \mathbf{r})=\frac{|x|^{\alpha_{1}-1}|y|^{\alpha_{2}-1}|z|^{\alpha_{3}-1}}{\Gamma\left(\alpha_{1}\right) \Gamma\left(\alpha_{2}\right) \Gamma\left(\alpha_{3}\right)},
$$

where $x, y, z$ are Cartesian's coordinates, and $D=\alpha_{1}+\alpha_{2}+\alpha_{3}, 0<D \leq 3$.

Note that for $D=2$, we have the fractal mass distribution in the volume. In general, this case is not equivalent to the distribution on the two-dimensional surface.

For $\rho(\mathbf{r})=\rho(|\mathbf{r}|)$, we can use the Riesz definition of the fractional integrals [1], and

$$
c_{3}(D, \mathbf{r})=\lambda(D)|\mathbf{r}|^{D-3}
$$

where

$$
\lambda(D)=\gamma_{3}^{-1}(D)=\frac{\Gamma(1 / 2)}{2^{D} \pi^{3 / 2} \Gamma(D / 2)} .
$$

Note that

$$
\lim _{D \rightarrow 3-} \gamma_{3}^{-1}(D)=\left(4 \pi^{3 / 2}\right)^{-1}
$$

Therefore, we suggest using

$$
\lambda(D)=\left(4 \pi^{3 / 2}\right) \gamma_{3}^{-1}(D)=\frac{2^{3-D} \Gamma(3 / 2)}{\Gamma(D / 2)} .
$$


The factor (46) allows us to derive the usual integral in the limit $D \rightarrow(3-0)$. Note that the final equations that relate mass, moment of inertia, and radius are independent of the numerical factor $\lambda(D)$.

For the homogeneous medium $\left(\rho(\mathbf{r})=\rho_{0}=\right.$ const $)$ and the ball region $W=\{\mathbf{r}:|\mathbf{r}| \leq R\}$,

$$
M_{D}(W)=\rho_{0} \frac{2^{3-D} \Gamma(3 / 2)}{\Gamma(D / 2)} \int_{W}|\mathbf{r}|^{D-3} d V_{3} .
$$

Using the spherical coordinates, we get

$$
\begin{aligned}
M_{D}(W)= & \frac{\pi 2^{5-D} \Gamma(3 / 2)}{\Gamma(D / 2)} \rho_{0} \int_{W}|\mathbf{r}|^{D-1} d|\mathbf{r}|= \\
& =\frac{2^{5-D} \pi \Gamma(3 / 2)}{D \Gamma(D / 2)} \rho_{0} R^{D} .
\end{aligned}
$$

As a result, we have $M(W) \sim R^{D}$, i.e., we derive the equation $M \sim R^{D}$ up to the numerical factor. Therefore the fractal medium with noninteger mass dimension $D$ can be described by fractional integral of order $D$.

\section{ELECTRODYNAMICS OF FRACTAL DISTRIBUTION OF CHARGED PARTICLES}

In this section, a brief review of electrodynamics of fractal distribution of charged particles [21] is considered to fix notation and provide a convenient reference.

\section{A. Electric charge for fractal distribution}

Let us consider charged particles that are distributed with a constant density over a fractal with Hausdorff dimension $D$. In this case, the electric charge $Q$ satisfies the scaling law $Q(R) \sim R^{D}$, whereas for a regular n-dimensional Euclidean object we have $Q(R) \sim R^{n}$.

The total charge of region $W$ is

$$
Q_{3}(W)=\int_{W} \rho(\mathbf{r}, t) d V_{3}
$$

where $\rho(\mathbf{r}, t)$ is a charge density in the region $W$. The fractional generalization of (47) is

$$
Q_{D}(W)=\int_{W} \rho(\mathbf{r}, t) d V_{D}
$$


where $D$ is a fractal dimension of the distribution, and

$$
d V_{D}=c_{3}(D, \mathbf{r}) d V_{3}
$$

The functions $c_{3}(D, \mathbf{r})$ is defined by the properties of the distribution.

If we consider the ball region $W=\{\mathbf{r}:|\mathbf{r}| \leq R\}$, and spherically symmetric distribution of charged particles $(\rho(\mathbf{r}, t)=\rho(r, t))$, then

$$
Q_{D}(R)=4 \pi \frac{2^{3-D} \Gamma(3 / 2)}{\Gamma(D / 2)} \int_{0}^{R} \rho(r) r^{D-1} d r
$$

For the homogeneous case, $\rho(r, t)=\rho_{0}$, and

$$
Q_{D}(R)=4 \pi \rho_{0} \frac{2^{3-D} \Gamma(3 / 2)}{\Gamma(D / 2)} \frac{R^{D}}{D} \sim R^{D} .
$$

The distribution of charged particles is called a homogeneous one if all regions $W$ and $W^{\prime}$ with the equal volumes $V_{D}(W)=V_{D}\left(W^{\prime}\right)$ have the equal total charges on these regions, $Q_{D}(W)=Q_{D}\left(W^{\prime}\right)$

\section{B. Electric current of fractal distribution}

For charged particles with density $\rho(\mathbf{r}, t)$ flowing with velocity $\mathbf{u}=\mathbf{u}(\mathbf{r}, t)$, the current density $\mathbf{J}(\mathbf{r}, t)$ is

$$
\mathbf{J}(\mathbf{r}, t)=\rho(\mathbf{r}, t) \mathbf{u}
$$

The electric current $I(S)$ is defined as the flux of electric charge. Measuring the field $\mathbf{J}(\mathbf{r}, t)$ passing through a surface $S=\partial W$ gives

$$
I(S)=\Phi_{J}(S)=\int_{S}\left(\mathbf{J}, d \mathbf{S}_{2}\right)
$$

where $d \mathbf{S}_{\mathbf{2}}=d S_{2} \mathbf{n}$ is a differential unit of area pointing perpendicular to the surface $S$, and the vector $\mathbf{n}=n_{k} \mathbf{e}_{k}$ is a vector of normal. The fractional generalization of (49) is

$$
I(S)=\int_{S}\left(\mathbf{J}(\mathbf{r}, t), d \mathbf{S}_{d}\right)
$$

where

$$
d S_{d}=c_{2}(d, \mathbf{r}) d S_{2}, \quad c_{2}(d, \mathbf{r})=\frac{2^{2-d}}{\Gamma(d / 2)}|\mathbf{r}|^{d-2} .
$$

Note that $c_{2}(2, \mathbf{r})=1$ for $d=2$. The boundary $\partial W$ has the dimension $d$. In general, the dimension $d$ is not equal to 2 and is not equal to $(D-1)$. 


\section{Charge conservation for fractal distribution}

The electric charge has a fundamental property established by numerous experiments: the velocity of charge change in region $W$ bounded by the surface $S=\partial W$ is equal to the flux of charge through this surface. This is known as the law of charge conservation:

$$
\frac{d Q(W)}{d t}=-I(S)
$$

or, in the form

$$
\frac{d}{d t} \int_{W} \rho(\mathbf{r}, t) d V_{D}=-\oint_{\partial W}\left(\mathbf{J}(\mathbf{r}, t), d \mathbf{S}_{d}\right) .
$$

In particular, when the surface $S=\partial W$ is fixed, we can write

$$
\frac{d}{d t} \int_{W} \rho(\mathbf{r}, t) d V_{D}=\int_{W} \frac{\partial \rho(\mathbf{r}, t)}{\partial t} d V_{D} .
$$

Using the fractional generalization of the Gauss's theorem (see the Appendix), we get

$$
\begin{gathered}
\oint_{\partial W}\left(\mathbf{J}(\mathbf{r}, t), d \mathbf{S}_{d}\right)= \\
=\int_{W} c_{3}^{-1}(D, \mathbf{r}) \frac{\partial}{\partial x_{k}}\left(c_{2}(d, \mathbf{r}) J_{k}(\mathbf{r}, t)\right) d V_{D} .
\end{gathered}
$$

The substitution of Eqs. (52) and (53) into Eq. (51) gives

$$
c_{3}(D, \mathbf{r}) \frac{\partial \rho(\mathbf{r}, t)}{\partial t}+\frac{\partial}{\partial x_{k}}\left(c_{2}(d, \mathbf{r}) J_{k}(\mathbf{r}, t)\right)=0 .
$$

As a result, we obtain the law of charge conservation in differential form (54). This equation can be considered as a continuity equation for fractal distribution of particles [11].

\section{Electric field and Coulomb's law}

For a point charge $Q$ at position $\mathbf{r}^{\prime}$, the electric field at a point $\mathbf{r}$ is defined by

$$
\mathbf{E}=\frac{Q}{4 \pi \varepsilon_{0}} \frac{\mathbf{r}-\mathbf{r}^{\prime}}{\left|\mathbf{r}-\mathbf{r}^{\prime}\right|^{3}}
$$

where $\varepsilon_{0}$ is a fundamental constant called the permittivity of free space.

For a continuous stationary distribution $\rho\left(\mathbf{r}^{\prime}\right)$,

$$
\mathbf{E}(\mathbf{r})=\frac{1}{4 \pi \varepsilon_{0}} \int_{W} \frac{\mathbf{r}-\mathbf{r}^{\prime}}{\left|\mathbf{r}-\mathbf{r}^{\prime}\right|^{3}} \rho\left(\mathbf{r}^{\prime}\right) d V_{3}^{\prime} .
$$


For Cartesian's coordinates $d V_{3}^{\prime}=d x^{\prime} d y^{\prime} d z^{\prime}$. The fractional generalization of (55) is

$$
\mathbf{E}(\mathbf{r})=\frac{1}{4 \pi \varepsilon_{0}} \int_{W} \frac{\mathbf{r}-\mathbf{r}^{\prime}}{\left|\mathbf{r}-\mathbf{r}^{\prime}\right|^{3}} \rho\left(\mathbf{r}^{\prime}\right) d V_{D}^{\prime},
$$

where $d V_{D}^{\prime}=c_{3}\left(D, \mathbf{r}^{\prime}\right) d V_{3}^{\prime}$. Equation (56) can be considered as Coulomb's law for a fractal stationary distribution of electric charges.

The electric field passing through a surface $S=\partial W$ gives the electric flux

$$
\Phi_{E}(S)=\int_{S}\left(\mathbf{E}, d \mathbf{S}_{2}\right)
$$

where $\mathbf{E}$ is the electric field vector, and $d \mathbf{S}_{2}$ is a differential unit of area pointing perpendicular to the surface S.

\section{E. Gauss's law for fractal distribution}

Gauss's law tells us that the total flux $\Phi_{E}(S)$ of the electric field $\mathbf{E}$ through a closed surface $S=\partial W$ is proportional to the total electric charge $Q(W)$ inside the surface:

$$
\Phi_{E}(\partial W)=\frac{1}{\varepsilon_{0}} Q(W) .
$$

For the fractal distribution, Gauss's law (57) states

$$
\int_{S}\left(\mathbf{E}, d \mathbf{S}_{2}\right)=\frac{1}{\varepsilon_{0}} \int_{W} \rho(\mathbf{r}, t) d V_{D}
$$

where $\mathbf{E}=\mathbf{E}(\mathbf{r}, t)$ is the electric field, and $\rho(\mathbf{r}, t)$ is the charge density, $d V_{D}=c_{3}(D, \mathbf{r}) d V_{3}$, and $\varepsilon_{0}$ is the permittivity of free space.

If $\rho(\mathbf{r}, t)=\rho(r)$, and $W=\{\mathbf{r}:|\mathbf{r}| \leq R\}$, then

$$
Q(W)=4 \pi \int_{0}^{R} \rho(r) c_{3}(D, \mathbf{r}) r^{2} d r
$$

where

$$
c_{3}(D, \mathbf{r})=\frac{2^{3-D} \Gamma(3 / 2)}{\Gamma(D / 2)}|\mathbf{r}|^{D-3} .
$$

Then

$$
Q(W)=4 \pi \frac{2^{3-D} \Gamma(3 / 2)}{\Gamma(D / 2)} \int_{0}^{R} \rho(r) r^{D-1} d r .
$$

For the sphere $S=\partial W=\{\mathbf{r}:|\mathbf{r}|=R\}$,

$$
\Phi_{E}(\partial W)=4 \pi R^{2} E(R)
$$


Substituting (60) and (61) in (57), we get

$$
E(R)=\frac{2^{3-D} \Gamma(3 / 2)}{\varepsilon_{0} R^{2} \Gamma(D / 2)} \int_{0}^{R} \rho(r) r^{D-1} d r .
$$

For homogeneous $(\rho(\mathbf{r})=\rho)$ distribution,

$$
E(R)=\rho \frac{2^{3-D} \Gamma(3 / 2)}{\varepsilon_{0} D \Gamma(D / 2)} R^{D-2} \sim R^{D-2} .
$$

\section{F. Magnetic field and Biot-Savart law}

The Biot-Savart law relates magnetic fields to the currents that are their sources. For a continuous distribution, the law is

$$
\mathbf{B}(\mathbf{r})=\frac{\mu_{0}}{4 \pi} \int_{W} \frac{\left[\mathbf{J}\left(\mathbf{r}^{\prime}\right), \mathbf{r}-\mathbf{r}^{\prime}\right]}{\left|\mathbf{r}-\mathbf{r}^{\prime}\right|^{3}} d V_{3}^{\prime},
$$

where [ , ] is a vector product, $\mathbf{J}$ is the current density, and $\mu_{0}$ is the permeability of free space. The fractional generalization of Eq. (62) is

$$
\mathbf{B}(\mathbf{r})=\frac{\mu_{0}}{4 \pi} \int_{W} \frac{\left[\mathbf{J}\left(\mathbf{r}^{\prime}\right), \mathbf{r}-\mathbf{r}^{\prime}\right]}{\left|\mathbf{r}-\mathbf{r}^{\prime}\right|^{3}} d V_{D}^{\prime} .
$$

This equation is the Biot-Savart law written for a steady current with a fractal distribution of electric charges. The law (63) can be used to find the magnetic field produced by any fractal distribution of steady currents.

\section{G. Ampere's law for fractal distribution}

The magnetic field in space around an electric current is proportional to the electric current that serves as its source. In the case of a static electric field, the line integral of the magnetic field around a closed loop is proportional to the electric current flowing through the loop. Ampere's law is equivalent to the steady state of the integral Maxwell equation in free space, and relates the spatially varying magnetic field $\mathbf{B}(\mathbf{r})$ to the current density $\mathbf{J}(\mathbf{r})$.

Note that, as mentioned in Ref. [19], Liouville, who was one of the pioneers in the development of fractional calculus, was inspired by the problem of fundamental force law in Ampbre's electrodynamics and used fractional differential equations in that problem.

Ampere's law states that the line integral of the magnetic field $\mathbf{B}$ along the closed path $L$ around a current given in MKS by

$$
\oint_{L}(\mathbf{B}, d \mathbf{l})=\mu_{0} I(S),
$$


where $d \mathbf{l}$ is the differential length element. For the distribution of particles on the fractal,

$$
I(S)=\int_{S}\left(\mathbf{J}, d \mathbf{S}_{d}\right)
$$

where $d \mathbf{S}_{d}=c_{2}(d, \mathbf{r}) d S_{2}$. For the cylindrically symmetric distribution,

$$
I(S)=2 \pi \int_{0}^{R} J(r) c_{2}(d, \mathbf{r}) r d r
$$

where $c_{2}(d, \mathbf{r})$ is defined in Eq. (150), i.e.,

$$
I(S)=4 \pi \frac{2^{2-d}}{\Gamma(d / 2)} \int_{0}^{R} J(r) r^{d-1} d r .
$$

For the circle $L=\partial W=\{\mathbf{r}:|\mathbf{r}|=R\}$, we get

$$
\oint_{L}(\mathbf{B}, d \mathbf{l})=2 \pi R B(R) .
$$

As a result,

$$
B(R)=\frac{\mu_{0} 2^{2-d}}{R \Gamma(d / 2)} \int_{0}^{R} J(r) r^{d-1} d r .
$$

For the homogeneous distribution, $J(r)=J_{0}$, and

$$
B(R)=J_{0} \frac{\mu_{0} 2^{2-d}}{d \Gamma(d / 2)} R^{d-1} \sim R^{d-1}
$$

\section{H. Fractional integral Maxwell equations}

Let us consider the fractional integral Maxwell equations [21]. The Maxwell equations are the set of fundamental equations for electric and magnetic fields. The equations that can be expressed in integral form are known as Gauss's law, Faraday's law, the absence of magnetic monopoles, and Ampere's law with displacement current. In MKS, these become

$$
\begin{gathered}
\oint_{S}\left(\mathbf{E}, d \mathbf{S}_{2}\right)=\frac{1}{\varepsilon_{0}} \int_{W} \rho d V_{D} \\
\oint_{L}\left(\mathbf{E}, d \mathbf{l}_{1}\right)=-\frac{\partial}{\partial t} \int_{S}\left(\mathbf{B}, d \mathbf{S}_{2}\right), \\
\oint_{S}\left(\mathbf{B}, d \mathbf{S}_{2}\right)=0 \\
\oint_{L}\left(\mathbf{B}, d \mathbf{l}_{1}\right)=\mu_{0} \int_{S}\left(\mathbf{J}, d \mathbf{S}_{d}\right)+\varepsilon_{0} \mu_{0} \frac{\partial}{\partial t} \int_{S}\left(\mathbf{E}, d \mathbf{S}_{2}\right) .
\end{gathered}
$$


Let us consider the fields that are defined on the fractal [40] only. The hydrodynamic and thermodynamics fields can be defined in the fractal media [11, 12]. Suppose that the electromagnetic field is defined on the fractal as an approximation of some real case with a fractal medium. If the electric and magnetic fields are defined on a fractal and does not exist outside of the fractal in Eucledian space $\mathbb{R}^{3}$, then we must use the fractional generalization of the integral Maxwell equations in the form [21]:

$$
\begin{gathered}
\oint_{S}\left(\mathbf{E}, d \mathbf{S}_{d}\right)=\frac{1}{\varepsilon_{0}} \int_{W} \rho d V_{D} \\
\oint_{L}\left(\mathbf{E}, d \mathbf{l}_{\gamma}\right)=-\frac{\partial}{\partial t} \int_{S}\left(\mathbf{B}, d \mathbf{S}_{d}\right) \\
\oint_{S}\left(\mathbf{B}, d \mathbf{S}_{d}\right)=0 \\
\oint_{L}\left(\mathbf{B}, d \mathbf{l}_{\gamma}\right)=\mu_{0} \int_{S}\left(\mathbf{J}, d \mathbf{S}_{d}\right)+\varepsilon_{0} \mu_{0} \frac{\partial}{\partial t} \int_{S}\left(\mathbf{E}, d \mathbf{S}_{d}\right) .
\end{gathered}
$$

Note that fractional integrals are considered as an approximation of integrals on fractals [38, 39].

Using the fractional generalization of Stokes's and Gauss's theorems (see the Appendix), we can rewrite Eqs. (64) in the form

$$
\begin{gathered}
\int_{W} c_{3}^{-1}(D, \mathbf{r}) d i v\left(c_{2}(d, \mathbf{r}) \mathbf{E}\right) d V_{D}=\frac{1}{\varepsilon_{0}} \int_{W} \rho d V_{D} \\
\int_{S} c_{2}^{-1}(d, \mathbf{r})\left(\operatorname{curl}\left(c_{1}(\gamma, \mathbf{r}) \mathbf{E}\right), d \mathbf{S}_{d}\right)=-\frac{\partial}{\partial t} \int_{S}\left(\mathbf{B}, d \mathbf{S}_{d}\right) \\
\int_{W} c_{3}^{-1}(D, \mathbf{r}) d i v\left(c_{2}(d, \mathbf{r}) \mathbf{B}\right) d V_{d}=0 \\
\int_{S} c_{2}^{-1}(d, \mathbf{r})\left(\operatorname{curl}\left(c_{1}(\gamma, \mathbf{r}) \mathbf{B}\right), d \mathbf{S}_{d}\right)=\mu_{0} \int_{S}\left(\mathbf{J}, d \mathbf{S}_{d}\right)+\varepsilon_{0} \mu_{0} \frac{\partial}{\partial t} \int_{S}\left(\mathbf{E}, d \mathbf{S}_{d}\right)
\end{gathered}
$$

As a result, we obtain

$$
\begin{gathered}
\operatorname{div}\left(c_{2}(d, \mathbf{r}) \mathbf{E}\right)=\frac{1}{\varepsilon_{0}} c_{3}(D, \mathbf{r}) \rho \\
\operatorname{curl}\left(c_{1}(\gamma, \mathbf{r}) \mathbf{E}\right)=-c_{2}(d, \mathbf{r}) \frac{\partial}{\partial t} \mathbf{B} \\
\operatorname{div}\left(c_{2}(d, \mathbf{r}) \mathbf{B}\right)=0 \\
\operatorname{curl}\left(c_{1}(\gamma, \mathbf{r}) \mathbf{B}\right)=\mu_{0} c_{2}(d, \mathbf{r}) \mathbf{J}+\varepsilon_{0} \mu_{0} c_{2}(d, \mathbf{r}) \frac{\partial \mathbf{E}}{\partial t} .
\end{gathered}
$$


Note that the law of absence of magnetic monopoles for the fractal leads us to the equation $\operatorname{div}\left(c_{2}(d, \mathbf{r}) \mathbf{B}\right)=0$. It can be rewritten as

$$
\operatorname{div} \mathbf{B}=-\left(\mathbf{B}, \operatorname{grad}_{2}(d, \mathbf{r})\right) .
$$

In general $(d \neq 2)$, the vector $\operatorname{grad}\left(c_{2}(d, \mathbf{r})\right)$ is not equal to zero and the magnetic field satisfies $\operatorname{div} \mathbf{B} \neq 0$. If $d=2$, we have $\operatorname{div}(\mathbf{B}) \neq 0$ only for nonsolenoidal field $\mathbf{B}$. Therefore the magnetic field on the fractal is similar to the nonsolenoidal field. As a result, the magnetic field on the fractal can be considered as a field with some "fractional magnetic monopole", $q_{m} \sim\left(\mathbf{B}, \nabla c_{2}\right)$.

\section{HYDRODYNAMICS OF FRACTAL MEDIA.}

\section{A. Euler equations for fractal media}

In Ref. [11], we derive the fractional generalizations of integral balance equations for fractal media. These equations leads to the following differential equations.

(1) The equation of continuity,

$$
\left(\frac{d}{d t}\right)_{D} \rho=-\rho \nabla_{k}^{D} u_{k}
$$

(2) The equation of balance of density of momentum,

$$
\rho\left(\frac{d}{d t}\right)_{D} u_{k}=\rho f_{k}+\nabla_{l}^{D} p_{k l}
$$

(3) The equation of balance of density of energy,

$$
\rho\left(\frac{d}{d t}\right)_{D} e=c(D, d, R) p_{k l} \frac{\partial u_{k}}{\partial x_{l}} .
$$

Here, we mean the sum on the repeated index, $k$ and $l$ from 1 to 3 , and use the notations

$$
\begin{gathered}
\nabla_{k}^{D} A=a(D, d) R^{3-D} \frac{\partial}{\partial x_{k}}\left(R^{d-2} A\right) \\
\left(\frac{d}{d t}\right)_{D}=\frac{\partial}{\partial t}+c(D, d, R) u_{l} \frac{\partial}{\partial x_{l}}= \\
=\frac{\partial}{\partial t}+a(D, d) R^{d+1-D} u_{l} \frac{\partial}{\partial x_{l}}
\end{gathered}
$$

where

$$
c(D, d, R)=a(D, d) R^{d+1-D},
$$




$$
a(D, d)=\frac{2^{D-d-1} \Gamma(D / 2)}{\Gamma(3 / 2) \Gamma(d / 2)} .
$$

The equations of balance are a set of five equations, which are not closed. These equations, in addition to the hydrodynamic fields $\rho(\mathbf{R}, t), u(\mathbf{R}, t), e(\mathbf{R}, t)$, include also the tensor of viscous stress $p_{k l}(\mathbf{R}, t)$. Let us consider the special cases of (65)-(67) with

$$
p_{k l}=-p \delta_{k l}
$$

where $p=p(\mathbf{R}, t)$ is the pressure. Then the hydrodynamics equations (65)-(67) are

$$
\begin{gathered}
\left(\frac{d}{d t}\right)_{D} \rho=-\rho \nabla_{k}^{D} u_{k} . \\
\left(\frac{d}{d t}\right)_{D} u_{k}=f_{k}-\frac{1}{\rho} \nabla_{k}^{D} p . \\
\left(\frac{d}{d t}\right)_{D} e=-c(D, d, R) \frac{p}{\rho} \frac{\partial u_{k}}{\partial x_{k}} .
\end{gathered}
$$

These equations are the Euler equations for the fractal medium.

\section{B. Equilibrium equation for fractal distribution}

The equilibrium state of medium means that

$$
\frac{\partial A}{\partial t}=0, \quad \frac{\partial A}{\partial x_{k}}=0
$$

for $A=\left\{\rho, u_{k}, e\right\}$. In this case, Eqs. (72)-(74) give

$$
f_{k}=\frac{1}{\rho} \nabla_{k}^{D} p
$$

Equation (75) gives the fractional generalization of the equilibrium equations. From (68), (70) and (71), Eq. (75) is

$$
\frac{\partial\left(c_{2}(d, R) p\right)}{\partial x_{k}}=\rho c_{3}(D, R) f_{k} .
$$

For the homogeneous medium $\rho(x)=$ const, and

$$
c_{3}(D, R) f_{k}=\frac{\partial\left(c_{2}(d, R) p / \rho_{0}\right)}{\partial x_{k}} .
$$

If $c_{3}(D, R) f_{k}=-\partial U / \partial x_{k}$, then

$$
c_{2}(d, R) p+\rho_{0} U=\text { const }
$$

This equation is a fractional generalization of the equilibrium equation. 


\section{Fractional Bernoulli integral}

Let us consider Eq. (73). Using Eq. (66) and

$$
\left(\frac{d}{d t}\right)_{D} \frac{\mathbf{u}^{2}}{2}=u_{k}\left(\frac{d}{d t}\right)_{D} u_{k}
$$

we get

$$
\left(\frac{d}{d t}\right)_{D} \frac{\mathbf{u}^{2}}{2}=u_{k} f_{k}-\frac{1}{\rho} u_{k} \nabla_{k}^{D} p .
$$

If

$$
\partial U / \partial t=0, \quad \partial p / \partial t=0
$$

then

$$
\left(\frac{d}{d t}\right)_{D}=c(D, d, R) \frac{d}{d t}
$$

Suppose

$$
f_{k}=-c(D, d, R) \partial U / \partial x_{k}
$$

If $D=3$ and $d=2$, then this force is potential. Using Eqs. (178) and (79), we get Eq. (777) in the form

$$
\frac{d}{d t}\left(\frac{\mathbf{u}^{2}}{2}+U+P(d)\right)=0
$$

where

$$
P(d)=\int_{p_{0}}^{p} \frac{d\left(c_{2}(d, R) p\right)}{c_{2}(d, R) \rho} .
$$

As a result, we obtain

$$
\sum_{k=1}^{3} \frac{u_{k}^{2}}{2}+U+P(d)=\text { const } .
$$

This integral of motion can be considered as a fractional generalization of the Bernoulli integral for fractal media. If the forces $f_{k}$ are potential, and $D \neq 3$, then the fractional analog of the Bernoulli integral does not exist.

For the density

$$
\rho=\rho_{0} c_{2}^{-1}(d, R)=\rho_{0} \frac{\Gamma(d / 2)}{2^{2-d}} R^{2-d}
$$

the integral (80) gives

$$
\frac{\rho_{0} \mathbf{u}^{2}}{2}+\rho_{0} U+c_{2}(d, R) p=\text { const }
$$

For $u_{k}=0$, Eq. (82) leads to Eq. (76). 


\section{Sound waves in fractal media}

Let us consider the small perturbations of Eqs. (72) and (73):

$$
\rho=\rho_{0}+\rho^{\prime}, \quad p=p_{0}+p^{\prime}, \quad u_{k}=u_{k}^{\prime},
$$

where $\rho^{\prime} \ll \rho_{0}$, and $p^{\prime} \ll p_{0}$, and $p_{0}$ and $\rho_{0}$ describe the steady state:

$$
\frac{\partial \rho_{0}}{\partial t}=0, \quad \frac{\partial \rho_{0}}{\partial x_{k}}=0, \quad \frac{\partial p_{0}}{\partial t}=0, \quad \frac{\partial p_{0}}{\partial x_{k}}=0 .
$$

Supposing $f_{k}=0$, and substituting (83) into Eqs. (72) and (73), we get

$$
\begin{aligned}
& \frac{\partial \rho^{\prime}}{\partial t}=-\rho_{0} \nabla_{k}^{D} u_{k}^{\prime}, \\
& \frac{\partial u_{k}^{\prime}}{\partial t}=-\frac{1}{\rho_{0}} \nabla_{k}^{D} p^{\prime} .
\end{aligned}
$$

To derive the independent equations for $\rho^{\prime}$, we consider the partial derivative of Eq. (84) with respect to time:

$$
\frac{\partial^{2} \rho^{\prime}}{\partial t^{2}}=-\rho \nabla_{k}^{D} \frac{\partial u_{k}^{\prime}}{\partial t} .
$$

The substitution of (85) into (86) obtains

$$
\frac{\partial^{2} \rho^{\prime}}{\partial t^{2}}=\nabla_{k}^{D} \nabla_{k}^{D} p^{\prime}
$$

For adiabatic processes $p=p(\rho, s)$, the first order of perturbation is

$$
p^{\prime}=v^{2} \rho^{\prime},
$$

where

$$
v=\sqrt{\left(\frac{\partial p}{\partial \rho}\right)_{s}} .
$$

As a result, we obtain

$$
\begin{aligned}
& \frac{\partial^{2} \rho^{\prime}}{\partial t^{2}}-v^{2} \nabla_{k}^{D} \nabla_{k}^{D} \rho^{\prime}=0, \\
& \frac{\partial^{2} p^{\prime}}{\partial t^{2}}-v^{2} \nabla_{k}^{D} \nabla_{k}^{D} p^{\prime}=0 .
\end{aligned}
$$

These equations describe the waves in the fractal medium. 


\section{MAGNETOHYDRODYNAMICS}

\section{A. Magnetohydrodynamics (MHD) equations}

The hydrodynamic and Maxwell equations for a fractal medium [11, 21] are the following.

(1) The equation of continuity,

$$
\left(\frac{d}{d t}\right)_{D} \rho=-\rho \nabla_{k}^{D} u_{k}
$$

(2) The equation of balance of density of momentum,

$$
\rho\left(\frac{d}{d t}\right)_{D} u_{k}=\rho f_{k}-\nabla_{k}^{D} p
$$

(3) Faraday's law,

$$
\operatorname{curl}\left(c_{1}(\gamma, \mathbf{r}) \mathbf{E}\right)=-c_{2}(d, \mathbf{r}) \frac{\partial}{\partial t} \mathbf{B}
$$

(4) The absence of magnetic monopoles

$$
\operatorname{div}\left(c_{2}(d, \mathbf{r}) \mathbf{B}\right)=0
$$

(5) Ampere's law,

$$
\operatorname{curl}\left(c_{1}(\gamma, \mathbf{r}) \mathbf{B}\right)=\mu_{0} c_{2}(d, \mathbf{r}) \mathbf{J}
$$

where the displacement current is neglected.

Using the Lorenz force density,

$$
\rho \mathbf{f}=[\mathbf{J}, \mathbf{B}]
$$

we get (91) in the form

$$
\rho\left(\frac{d}{d t}\right)_{D} \mathbf{u}+\nabla^{D} p=[\mathbf{J}, \mathbf{B}] .
$$

We assume a linear relationship between the $\mathbf{J}$ and $\mathbf{E}_{*}$ :

$$
\mathbf{J}(\mathbf{r}, t)=\sigma \mathbf{E}_{*}(\mathbf{r}, t)
$$

where $\sigma$ is the electric conductivity, and $\mathbf{E}_{*}$ is an electric field in the moved coordinate system. For $|\mathbf{u}| \ll c$,

$$
\mathbf{E}=\mathbf{E}_{*}-\frac{1}{c}[\mathbf{u}, \mathbf{B}]
$$

From (97), and (94), we get

$$
\mathbf{E}_{*}=\sigma^{-1} \mathbf{J}=\frac{1}{\sigma \mu_{0} c_{2}(d, \mathbf{r})} \operatorname{curl}\left(c_{1}(\gamma, \mathbf{r}) \mathbf{B}\right) .
$$


Substitution of (98) into (92) gives

$$
\begin{gathered}
\operatorname{curl}\left(c_{1}(\gamma, \mathbf{r}) \mathbf{E}_{*}-c_{1}(\gamma, \mathbf{r}) \frac{1}{c}[\mathbf{u}, \mathbf{B}]\right)= \\
=-c_{2}(d, \mathbf{r}) \frac{\partial}{\partial t} \mathbf{B} .
\end{gathered}
$$

Substituting (99) into (100), we have

$$
\begin{aligned}
& \operatorname{curl}\left(\frac{c_{1}(\gamma, \mathbf{r})}{\sigma \mu_{0} c_{2}(d, \mathbf{r})} \operatorname{curl}\left(c_{1}(\gamma, \mathbf{r}) \mathbf{B}\right)-\right. \\
& \left.-c_{1}(\gamma, \mathbf{r}) \frac{1}{c}[\mathbf{u}, \mathbf{B}]\right)=-c_{2}(d, \mathbf{r}) \frac{\partial}{\partial t} \mathbf{B} .
\end{aligned}
$$

Then,

$$
\begin{aligned}
c_{2}(d, \mathbf{r}) \frac{\partial}{\partial t} \mathbf{B}= & -\operatorname{curl}\left(\frac{c_{1}(\gamma, \mathbf{r})}{\sigma \mu_{0} c_{2}(d, \mathbf{r})} \operatorname{curl}\left(c_{1}(\gamma, \mathbf{r}) \mathbf{B}\right)\right)+ \\
& +\operatorname{curl}\left(c_{1}(\gamma, \mathbf{r}) \frac{1}{c}[\mathbf{u}, \mathbf{B}]\right) .
\end{aligned}
$$

As a result, we obtain magnetohydrodynamics (MHD) equations for a fractal distribution of charged particles:

(1) The equation of continuity,

$$
\left(\frac{d}{d t}\right)_{D} \rho=-\rho \nabla^{D} \mathbf{u}
$$

(2) The equation of balance of density of momentum,

$$
\rho\left(\frac{d}{d t}\right)_{D} \mathbf{u}+\nabla^{D} p=[\mathbf{J}, \mathbf{B}]
$$

(3) The absence of magnetic monopoles,

$$
\operatorname{div}\left(c_{2}(d, \mathbf{r}) \mathbf{B}\right)=0
$$

(4) The Ampere law,

$$
\operatorname{curl}\left(c_{1}(\gamma, \mathbf{r}) \mathbf{B}\right)=\mu_{0} c_{2}(d, \mathbf{r}) \mathbf{J}
$$

(5) The diffusion equation for the magnetic field,

$$
\begin{aligned}
c_{2}(d, \mathbf{r}) \frac{\partial}{\partial t} \mathbf{B}= & -\operatorname{curl}\left(\frac{c_{1}(\gamma, \mathbf{r})}{\sigma \mu_{0} c_{2}(d, \mathbf{r})} \operatorname{curl}\left(c_{1}(\gamma, \mathbf{r}) \mathbf{B}\right)\right)+ \\
& +\operatorname{curl}\left(c_{1}(\gamma, \mathbf{r}) \frac{1}{c}[\mathbf{u}, \mathbf{B}]\right) .
\end{aligned}
$$

We have 11 equations for 11 variables $p, \rho, \mathbf{J}, \mathbf{u}$, and $\mathbf{B}$. 


\section{B. Equilibrium from MHD equations}

Let us consider the stationary (equilibrium) states for MHD equations. The total time derivatives in Eqs. (104) and (106) are equal to zero, and

$$
\begin{gathered}
\nabla^{D} p=[\mathbf{J}, \mathbf{B}], \\
\mathbf{J}=\frac{1}{\mu_{0} c_{2}(d, \mathbf{r})} \operatorname{curl}\left(c_{1}(\gamma, \mathbf{r}) \mathbf{B}\right) .
\end{gathered}
$$

The substitution of (109) into (110) obtains

$$
\nabla^{D} p=\frac{1}{\mu_{0} c_{2}(d, \mathbf{r})}\left[\operatorname{curl}\left(c_{1}(\gamma, \mathbf{r}) \mathbf{B}\right), \mathbf{B}\right] .
$$

Suppose $\mathbf{B}=\left\{0,0, B_{z}\right\}$. Then

$$
\begin{gathered}
\operatorname{curl}\left(c_{1}(\gamma, \mathbf{r}) \mathbf{B}\right)= \\
=\mathbf{e}_{x} \partial_{y}\left(c_{1}(\gamma, \mathbf{r}) B_{z}\right)-\mathbf{e}_{y} \partial_{x}\left(c_{1}(\gamma, \mathbf{r}) B_{z}\right),
\end{gathered}
$$

and

$$
\begin{gathered}
{\left[\operatorname{curl}\left(c_{1}(\gamma, \mathbf{r}) \mathbf{B}\right), \mathbf{B}\right]=} \\
=-\mathbf{e}_{x} B_{z} \partial_{x}\left(c_{1}(\gamma, \mathbf{r}) B_{z}\right)-\mathbf{e}_{y} B_{z} \partial_{y}\left(c_{1}(\gamma, \mathbf{r}) B_{z}\right) .
\end{gathered}
$$

As a result, Eq. (108) gives

$$
\begin{aligned}
& \nabla_{x}^{D} p=-\frac{1}{\mu_{0} c_{2}(d, \mathbf{r})} B_{z} \partial_{x}\left(c_{1}(\gamma, \mathbf{r}) B_{z}\right), \\
& \nabla_{y}^{D} p=-\frac{1}{\mu_{0} c_{2}(d, \mathbf{r})} B_{z} \partial_{y}\left(c_{1}(\gamma, \mathbf{r}) B_{z}\right) .
\end{aligned}
$$

From the definition of $\nabla^{D}$, we have

$$
\begin{gathered}
\frac{\partial}{\partial x} c_{2}(d, \mathbf{r}) p=-\frac{c_{3}(D, \mathbf{r})}{\mu_{0} c_{2}(d, \mathbf{r})} B_{z} \frac{\partial}{\partial x}\left(c_{1}(\gamma, \mathbf{r}) B_{z}\right) \\
\frac{\partial}{\partial y} c_{2}(d, \mathbf{r}) p=-\frac{c_{3}(D, \mathbf{r})}{\mu_{0} c_{2}(d, \mathbf{r})} B_{z} \frac{\partial}{\partial y}\left(c_{1}(\gamma, \mathbf{r}) B_{z}\right) .
\end{gathered}
$$

Using $A \partial B=\partial(A B)-B \partial A$, we get

$$
\begin{aligned}
& \frac{\partial}{\partial x}\left(c_{2}(d, \mathbf{r}) p+\frac{c_{3}(D, \mathbf{r}) c_{1}(\gamma, \mathbf{r})}{\mu_{0} c_{2}(d, \mathbf{r})} B_{z}^{2}\right)= \\
& \quad=c_{1}(\gamma, \mathbf{r}) B_{z} \frac{\partial}{\partial x}\left(\frac{c_{3}(D, \mathbf{r})}{\mu_{0} c_{2}(d, \mathbf{r})} B_{z}\right)
\end{aligned}
$$




$$
\begin{gathered}
\frac{\partial}{\partial y}\left(c_{2}(d, \mathbf{r}) p+\frac{c_{3}(D, \mathbf{r}) c_{1}(\gamma, \mathbf{r})}{\mu_{0} c_{2}(d, \mathbf{r})} B_{z}^{2}\right)= \\
=c_{1}(\gamma, \mathbf{r}) B_{z} \frac{\partial}{\partial y}\left(\frac{c_{3}(D, \mathbf{r})}{\mu_{0} c_{2}(d, \mathbf{r})} B_{z}\right) \\
\frac{\partial}{\partial y}\left(c_{2}(d, \mathbf{r}) p\right)=0
\end{gathered}
$$

As a result, we obtain

$$
c_{2}(d, \mathbf{r}) p+\frac{c_{3}(D, \mathbf{r}) c_{1}(\gamma, \mathbf{r})}{\mu_{0} c_{2}(d, \mathbf{r})} B_{z}^{2}=\text { const }
$$

This equilibrium equation exists only if

$$
B_{z} \sim \frac{\mu_{0} c_{2}(d, \mathbf{r})}{c_{3}(D, \mathbf{r})}
$$

It is easy to see that we do not have the usual invariants for the fractal distribution of charged particles. Therefore equilibrium on the fractal exists for the magnetic field that satisfies the power law relation

$$
B_{z} \sim R^{d-D+1}
$$

For the distribution with an integer Hausdorff dimension, we have the usual relation [42]. The typical turbulent media could be of fractal structure, and the corresponding equations should be changed to include the fractal features of the media. Therefore, the equilibrium of the fractal turbulent medium exists for the magnetic field with the power law relation (119).

\section{CONCLUSION}

Typical turbulent media could be of a fractal structure, and the corresponding equations should be changed to include the fractal features of the media. Magnetohydrodynamics equations for the fractal distribution of charged particles are suggested. The fractional integrals are used to describe fractal distribution. These integrals are considered as approximations of integrals on fractals. Using the fractional generalization of the integral Maxwell equation and the integral balance equations, we derive the magnetohydrodynamics equations. Equilibrium states for these equations are discussed. The equilibrium for fractal turbulent media can exists if the magnetic field satisfies the power law relation. 


\section{APPENDIX: FRACTIONAL GAUSS'S THEOREM}

Let us derive the fractional generalization of Gauss's theorem,

$$
\int_{\partial W}\left(\mathbf{J}(\mathbf{r}, t), d \mathbf{S}_{2}\right)=\int_{W} \operatorname{div}(\mathbf{J}(\mathbf{r}, t)) d V_{3},
$$

where the vector $\mathbf{J}(\mathbf{r}, t)=J_{k} \mathbf{e}_{k}$ is a field, and $\operatorname{div}(\mathbf{J})=\partial \mathbf{J} / \partial \mathbf{r}=\partial J_{k} / \partial x_{k}$. Here, we mean the sum on the repeated index $k$ from 1 to 3 . Using

$$
d \mathbf{S}_{d}=c_{2}(d, \mathbf{r}) d \mathbf{S}_{2}, \quad c_{2}(d, \mathbf{r})=\frac{2^{2-d}}{\Gamma(d / 2)}|\mathbf{r}|^{d-2},
$$

we get

$$
\int_{\partial W}\left(\mathbf{J}(\mathbf{r}, t), d \mathbf{S}_{d}\right)=\int_{\partial W} c_{2}(d, \mathbf{r})\left(\mathbf{J}(\mathbf{r}, t), d \mathbf{S}_{2}\right) .
$$

Note that $c_{2}(2, \mathbf{r})=1$ for $d=2$. Using (120), we get

$$
\int_{\partial W} c_{2}(d, \mathbf{r})\left(\mathbf{J}(\mathbf{r}, t), d \mathbf{S}_{2}\right)=\int_{W} \operatorname{div}\left(c_{2}(d, \mathbf{r}) \mathbf{J}(\mathbf{r}, t)\right) d V_{3} .
$$

The relation

$$
d V_{D}=c_{3}(D, \mathbf{r}) d V_{3}, \quad c_{3}(D, \mathbf{r})=\frac{2^{3-D} \Gamma(3 / 2)}{\Gamma(D / 2)}|\mathbf{r}|^{D-3}
$$

in the form $d V_{3}=c_{3}^{-1}(D, \mathbf{r}) d V_{D}$ allows us to derive the fractional generalization of Gauss's theorem:

$$
\begin{gathered}
\int_{\partial W}\left(\mathbf{J}(\mathbf{r}, t), d \mathbf{S}_{d}\right)= \\
=\int_{W} c_{3}^{-1}(D, \mathbf{r}) \operatorname{div}\left(c_{2}(d, \mathbf{r}) \mathbf{J}(\mathbf{r}, t)\right) d V_{D} .
\end{gathered}
$$

Analogously, we can get the fractional generalization of Stokes's theorem in the form

$$
\oint_{L}\left(\mathbf{E}, d \mathbf{l}_{\gamma}\right)=\int_{S} c_{2}^{-1}(d, \mathbf{r})\left(\operatorname{curl}\left(c_{1}(\gamma, \mathbf{r}) \mathbf{E}\right), d \mathbf{S}_{d}\right),
$$

where

$$
c_{1}(\gamma, \mathbf{r})=\frac{2^{1-\gamma} \Gamma(1 / 2)}{\Gamma(\gamma / 2)}|\mathbf{r}|^{\gamma-1}
$$

[1] S. G. Samko, A. A. Kilbas, O. I. Marichev, Fractional Integrals and Derivatives Theory and Applications (Gordon and Breach, New York, 1993)

[2] K.B. Oldham, J. Spanier, The Fractional Calculus (Academic Press, New York, 1974) 
[3] G.M. Zaslavsky, Phys. Rep. 371, 461 (2002).

[4] G.M. Zaslavsky, Hamiltonian Chaos and Fractional Dynamics (Oxford University Press, Oxford, 2005)

[5] R. Hilfer (Ed.), Applications of Fractional Calculus in Physics (World Scientific, Singapore, 2000)

[6] M. Caputo, Elasticita e Dissipazione (Zanichelli, Bologna, 1969) in Italy

[7] R.R. Nigmatullin, Phys. Status Solidi B 133, 425 (1986); Theor. Math. Phys. 90, 242 (1992).

[8] A. Le Mehaute, R.R. Nigmatullin, L. Nivanen, Fleches du temps et geometric fractale (Hermes, Paris, 1998) Chapter 5. in French

[9] A. Carpinteri, F. Mainardi, Fractals and Fractional Calculus in Continuum Mechanics (Springer, New York, 1997)

[10] V.E. Tarasov, Phys. Lett. A 336, 167 (2005); Chaos 15, 023102 (2005); Phys. Lett. A 341, 467 (2005); Mod. Phys. Lett. B 19, 721 (2005).

[11] V.E. Tarasov, Ann. Phys. 318, 286 (2005).

[12] V.E. Tarasov, G.M. Zaslavsky, Physica A 354, 249 (2005).

[13] N. Laskin, Phys. Rev. E 66, 056108 (2002); Chaos 10, 780 (2000); Phys. Rev. E 62, 3135 (2000); Phys. Lett. A 268, 298 (2000).

[14] M. Naber, J. Math. Phys. 45, 3339 (2004).

[15] G.M. Zaslavsky, Physica D 76, 110 (1994).

[16] A.I. Saichev, G.M. Zaslavsky, Chaos 7, 753 (1997).

[17] G.M. Zaslavsky, M.A. Edelman, Physica D 193, 128 (2004).

[18] B.A. Carreras, V.E. Lynch, G.M. Zaslavsky, Physics of Plasmas 8, 5096 (2001).

[19] J. Lutzen, "Liouville's differential calculus of arbitrary order and its electrodynamical origin," in Proc. 19th Nordic Congress Mathenzaticians, (Icelandic Mathematical Soc., Reykjavik, 1985) pp.149-160.

[20] L.M. Zelenyi, A.V. Milovanov, Physics Uspekhi 47, 749 (2004).

[21] V.E. Tarasov, Physics of Plasmas 12, 082106 (2005); Mod. Phys. Lett. B. 19, 1107 (2005).

[22] V.E. Tarasov, Celestial Mech. Dynam. Astron. 19, 1 (2006).

[23] F. Mainardi, R. Gorenflo, J. Comput. Appl. Math. 118, 283 (2000).

[24] V.E. Tarasov, G.M. Zaslavsky "Dynamics with low-level fractionality" Physica A. 368, 399 (2006). 
[25] V.E. Tarasov, Chaos 14, 123 (2004); Phys. Rev. E 71, 011102 (2005); J. Phys. Conf. Ser. 7, $17(2005)$.

[26] V.E. Tarasov, J. Phys. A 38, 5929 (2005); Lett. Math. Phys. 73, 49 (2005).

[27] N. Laskin, G.M. Zaslavsky, Physica A 368, 38 (2006).

[28] V.E. Tarasov, G.M. Zaslavsky, Chaos 16, 023110 (2006).

[29] E.W. Montroll, M.F. Shlesinger, "The wonderful world of random walks" In: Studies in Statistical Mechanics, Vol. 11. J. Lebowitz, E. Montroll (Eds.), (North-Holland, Amsterdam, 1984) pp.1-121.

[30] V.V. Uchaikin, Physics-Uspekhi 46, 821 (2003); J. Exper. Theor. Phys. 97, 810 (2003).

[31] R. Metzler, J. Klafter, Phys. Rep. 339, 1 (2000).

[32] H. Federer, Geometric Measure Theory (Berlin, Springer, 1969)

[33] C.A. Rogers, Hausdorff Measures, 2nd ed. (Cambridge, Cambridge University Press, 1999).

[34] G.A. Edgar, Measure, Topology, and Fractal Geometry (New York: Springer-Verlag, 1990).

[35] K.F. Falconer, The Geometry of Fractal Sets (Cambridge, Cambridge University Press, 1985)

[36] K.G. Wilson, Phys. Rev. D 7, 2911 (1973).

[37] J.C. Collins, Renormalization (Cambridge University Press, Cambridge, 1984) Sec. 4.

[38] K. Svozil, J. Phys. A 20, 3861 (1987).

[39] F.Y. Ren, J.R. Liang, X.T. Wang, W.Y. Qiu, Chaos, Solit. Fract. 16, 107 (2003).

[40] J. Feder, Fractals (Plenum Press, New York, 1988) Sec. 5 and 6.

[41] R.M. Christensen, Mechanics of Composite Materials (Wiley, New York, 1979).

[42] B.B. Kadomtsev, Collective Phenomena in Plasmas (Pergamon, New York, 1978) 